\title{
Energy Efficient Routing Protocol BASEd ON DSR
}

\author{
Jijesh J. J and Shivashankar \\ Department of Electronics \& Communication Engineering, \\ Sri Venkateshwara College of Engineering, VTU, Bangalore, India
}

\begin{abstract}
Energy consumption is a major concern in most of the present day devices in wireless networks. Especially in Ad hoc networks, energy is a limited factor. Random movement in nodes add to the frequent failure of routes which adds to the energy consumption in the network. In this paper, a routing protocol is proposed which is based on a modification of the conventional DSR (Dynamic Source routing). A comparative analysis is performed with respect to energy consumption, maximum throughput and delay. The routing protocols used for reference in this analysis are DSDV, AODV and conventional DSR. Experimental results show that the proposed modified DSR shows a reduced energy consumption, improved rate of maximum throughut and a reduced delay compared to above mentioned routing protocols.
\end{abstract}

\section{KEYWORDS}

Dynamic Source Routing (DSR), DSDV, Ad hoc networks and On-Demand Routing Protocols.

\section{INTRODUCTION}

In many of the modern day applications concerning the interconnectivity between devices, or what we may term it as smart devices play a significant role in many sectors ranging from military to healthcare applications. An example for such an application is the Wireless Body Area Network (WBAN) which are defined as medical sensors (monitoring devices) used for detecting and measuring the physiological activities produced by human beings from Brain, Heart, etc. which is measured by metrics such as Electroencephalogram, (EEG) Electrocardiogram (ECG),etc. . Here, considering more number of patients with more monitoring devices creates a proportionally complex network of various monitoring devices along with patients. Therefore, the process of efficient routing between the devices becomes a very crucial part in the seemingly overall complex network structure. Hence, there is a need to create a more efficient as well as robust routing protocols (RP) in the case of complex network structures. A general classification of RP is given in figure 1 .

One of the many issues concerning the design aspects in RPs is that related to energy consumption. Considering the energy in the static based RP which basically involves manual entering and administering the routing table as compared to dynamic based RP where the routing table is updated dynamically without manual interference, there is a much higher efficiency in the latter RP [1].

Moez Esseghir [2] performed an implementation on energy based RP having dynamic routing in wireless sensor networks. Experimental results demonstrated an increase in the network lifetime which was prevalent in dynamic based RP as compared to Static RP.K. Srinivas et. al. [3] addresses the issue concerning the overall network lifetime by signifying the role of energy consumption in three different types of RP in both static along with dynamic Ad hoc networks. The RP's considered were Ad hoc on-demand Distance Vector routing (AODV), Dynamic Source Routing (DSR) and Wireless Routing Protocol (WRP). A performance analysis on the 
same signified that in the case of mobile Ad hoc networks, the energy efficiency was better in DSR as compared to AODV and WRP.

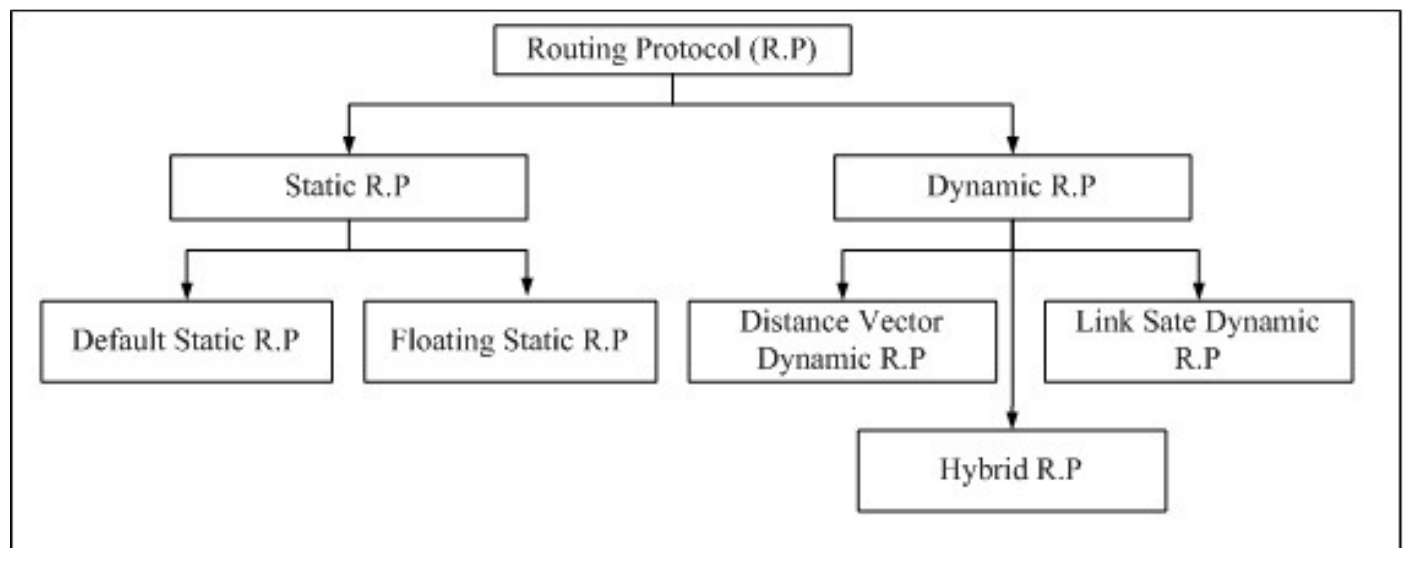

Figure 1: General classification of RP

The standard routing protocols which are considered for implementation is mentioned below as follows [4].

1. Distance Vector Routing Protocol (DVRP)

This type of routing protocols calculates a cumulative distance between routers which is based on distance metrics such as the Bell-Ford algorithm, Ford Fulkerson algorithm or DUAL (Diffusing Update ALgorithm) FSM. Some of the distance vector routing protocols include RIPv1 (Routing Information Protocol) and RIPv2.

2. Link State Routing protocols

This type of protocol maintains a complex database comprising of internetwork topology. However, the algorithms of this kind of protocol depend on the state of the link between the routers. Examples of this type of protocols contain Open Shortest Path First (OSPF) and Intermediate System to Intermediate System (IS-IS)

3. Hybrid Routing protocols

This kind of routing protocols comprises of a combination of the above two protocols. The significance of this type of routing protocols is that it allows for quick convergence but requires a lesser amount of processing memory and power when compared with link state routing protocol. An example for a hybrid protocol is the Enhance Interior Gateway Routing Protocol (EIGRP) which was developed by Cisco Systems, Inc.

The paper is organized as follows. The first section briefly illustrates the issues and its relevant techniques which were applied. The second section performs a review of literature with respect to issues and respective techniques applied to address those issues. The third section explains the proposed system involving different modules and their respective functions. The fourth section performs an evaluation of the proposed method. The paper is concluded with a last section providing a conclusion of the overall experiment.

\section{RELATED WORKS}

One of the conventions considered for the decision and transmission of route packets in a particular route is ad hoc routing protocols. The general types concerning the ad hoc routing 
protocol are table driven (Proactive) routing protocol, On-demand (Reactive) routing protocol and the hybrid routing protocol (which includes both proactive and reactive routing protocol).

M. Bharathi et. al [5] performed an evaluation with respect to energy consumption for AODV and DSR routing protocols within Mobile ad hoc network (MANET). By the consideration of random Waypoint Mobility (RWM) model. The performance with respect to energy metrics was evaluated. The parameters used for this evaluation were traffic pattern, mobility pattern of the node, number of mobile nodes as well as the simulation area size. Experimental results indicate that energy consumption could be decreased along with an increase in the network lifetime by reducing number of control packets along with considering the routing overhead of AODV protocol.

K. Kunavut [6] performed a comparative study on energy consumption and Mobile Ad hoc routing protocol. The standardized routing protocols considered to obtain the best paths were Internet Engineering Task Force (IETF), MANET working group such as OLSR (RFC3626), DSR (RFC 4728) and AODV (RFC3561). The total energy consumption along with energy consumed at every state with respect to standard routing protocols are compared, measured and analyzed. Simulation results show that the OLSR has the least energy consumption in scenarios considering the speed, load and node varying situation. However, AODV and DSR have approximately same intensity of energy consumption with respect to load and node varying conditions.

S. Alam et. al [7] addresses the factor of energy consumption and performs an analysis for the same considering the IARP, RIP and STAR routing protocols concerning wireless sensor networks. The simulator used in this environment is QualNet 7.1 for performing simulation analysis with respect to IARP, Rip and STAR routing protocols. The performance metrics considered in this experiment are number of revised packets transmitted, node wise energy consumption and average energy which is consumed during transmission, receiving and idle state. The simulation results show an increased transmission rate with respect to number of update packets in IARP. Performance of RIP is much better in both transmission and receiving state. Hence, it was observed that RIP had a better energy efficiency compared to IARP and STAR topology in transmission and receiving state.

E. Tavosian et. al [8] projected a method for improving the energy consumption in AODV routing protocol using network coding. A hop to hop coding technique is used in this experiment which is perceived to be a conventional IP forwarding mechanism. The outcome of this experiment was a modified AODV scheme which consumes less energy along with reducing the number of data transmission.

H. Kajikawa et. al [9] proposes a grid based routing protocol for the purpose of reduction of packet latency and energy consumption. The area of network is divided for side length of square cell using the technique of cell rotation which reduces number of relay nodes between source node and mobile sink. This inturn reduces the latency of packet data delivery and energy consumption.

C.C Weng et. al [10] proposed a new routing protocol for minimization of energy consumption during the transmission stage considering the user centric wireless network. The respective protocol is based on various factors such as data transmission, channel events, node energy and traffic loads involving nodes. This protocol also signifies a reduced energy consumption along with increased network lifetime compared to other standard protocols.

H. Qin et. al [11] proposed a cluster based routing protocol with a view to balance energy consumption. The most significant clustering based routing protocol is the LEACH protocol (Low Energy Adaptive Clustering hierarchy) which has been modified in this work for the 
purpose of monitoring large scale environment for a stable and longer duration of time. During the time of transmission of packets the path constructed by gateways is multi hop based. Experimental result shows an improved balance in energy consumption and network lifetime.

J. Friginal et. al [12] performs an evaluation of different routing protocols for Ad hoc networks with respect to performance and energy consumption. The work includes an assessment of fault prevention of encryption and detection mechanism for olsrd routing protocols. The experimental outcome explores the effect on energy consumption of different routing protocols with regard to malicious and accidental faults.

J.H Zhang et. al [13] proposed a MANET based routing protocols considering the mobility models for the purpose of analysis of energy consumption. This work introduces an evaluation of energy consumption based on bytes. Experimental results show an increased efficiency in energy consumption with protocols for example AODV, DSR and DSDV in MANET at high node mobility conditions.

G. Singh Brar et. al [14] proposed energy efficient routing protocol based on direction based PDORP with respect to wireless sensor network. It is considered that the DSR routing protocol is more efficient when it comes to density of energy in small scale, But at certain instance of time the efficiency decreases when the node changes to sleep mode from active mode. As a result there is a raise in end-to-end delay as well as an increase in waiting time. The overall consequence leads to an increase in consumption of energy in the network. In order to find the optimal path a hybrid technique involving for aging optimization with GA (Genetic Algorithm) is applied. Experimental results showed a reduced bit error rate along with reduction in delay, energy consumption and an improved throughput resulting in an improved network lifetime.

L. Pan [15] proposed an improved routing protocol based on mobile ad hoc network. By acquiring steady routing responses of contagious nodes, less mobility is achieved with resource nodes. Proposed routing protocol in this work signifies an improved efficiency with reduced used of network resources which is limited. Simulation result shows an improved rate compared to DSR in terms of routing length, discovery time, routing error and propagating requests.

Ganesh R. Pathak et. al [16] proposed a mathematical model for routing protocol performing a comparative analysis on DSR, AODV and DSDV. The work also presented a pre-simulation tcl file for the purpose of performance evaluation. A comparative analysis is performed on two ondemand routing protocol which are DSR and AODV along with one table driven routing protocol DSDV. Experimental results show that the DSR has a better performance as compared to other protocols.

R. Ahuja et. al [17] performed a comparative analysis on AODV and DSR routing protocols in connection with MANET in a worm hole attack. This work has considered the security issues concerning the wireless networks. In various attacks which are prevalent in routing protocol, one such attack is the wormhole attack. AN evaluation of performance with respect to AODV and DSR is performed considering the worm hole attack. Experimental results show an increase in performance efficiency in DSR routing protocol.

A.B Tambuwal et. al [18] proposed a ttl based DSR routing protocol in mobile ad hoc networks. This work performs an investigation on DSDV and conventional DSR routing protocol. It was observed that when the nodes had high mobility the DSDV performed better than the DSR routing protocol. In this context a ttl based DSR was proposed with an intention to improve its performance. Experimental outcome explains that the proposed technique showed an improvement in the network throughput. 
A general statistics on the research work carried out in energy consumption and DSR routing protocol are given in table 1 and table 2 respectively as shown below. (Note: The content was procured from IEEE digital library at $3^{\text {rd }}$ September, 2016 at IST 7:43 am). The contents include Conferences, publications, books, Journals, etc.

Table 1: statistics on research work concerning energy consumption

\begin{tabular}{|l|l|l|}
\hline Sl.no & \multicolumn{1}{|c|}{ Type of publications } & \multicolumn{1}{c|}{ volume } \\
\hline \multicolumn{1}{|c|}{ Year : 1992 - 2016 } & 15,425 \\
\hline 1. & Conference Publications & 2,202 \\
\hline 2. & Journals and magazines & 113 \\
\hline 3. & Publication title & 10 \\
\hline 4. & Standards & 6 \\
\hline 5. & Books and EBooks & \\
\hline 6. & Publisher & 17,381 \\
& a. IEEE & 276 \\
& b. IET & 77 \\
& c. VDE & 8 \\
& d. BIAI & 8 \\
& e. TUP & 3 \\
& f. Alcatel - Lucent & 1 \\
& g. IBM & 1 \\
& h. MITP & 1 \\
\hline
\end{tabular}

Table 2: statistics on research work concerning DSR routing protocol

\begin{tabular}{|c|l|l|}
\hline Sl.no & Type of publications & volume \\
\hline \multicolumn{2}{|l|}{ Year : 1998-2016 } \\
\hline 1. & Conference Publications & 1,180 \\
\hline 2. & Journals and magazines & 49 \\
\hline 3. & Publication title & 25 \\
\hline 4. & Publisher & \\
& a. IEEE & 1,197 \\
& b. IET & 28 \\
& c. VDE & 2 \\
& d. BIAI & 1 \\
& e. TUP & 1 \\
\hline
\end{tabular}

\section{DynAMic Source Routing}

Dynamic Source Routing (DSR) is route on-demand type of routing protocol which is very much similar to AODV. However, DSR does not rely on routing table unlike AODV but instead uses source routing at each intermediate device [19]. The DSR is primarily designed to limit the usage of bandwidth which is consumed by control packets present in Ad hoc networks. During the phase of route construction, it establishes a route by broadcasting route request packet in the network. The destination node upon receiving the respective route request packet sends a response in the form of RouteReply (RREP) packet to the source node carrying the route travelled by RouteRequest (RREQ) Packet received. The significance of DSR mainly in reactive approach is that it eliminates the necessity of periodical broadcasting in the network which is predominant in table driven approach [20]. The algorithm for route discovery and route maintenance is given below as follows. 

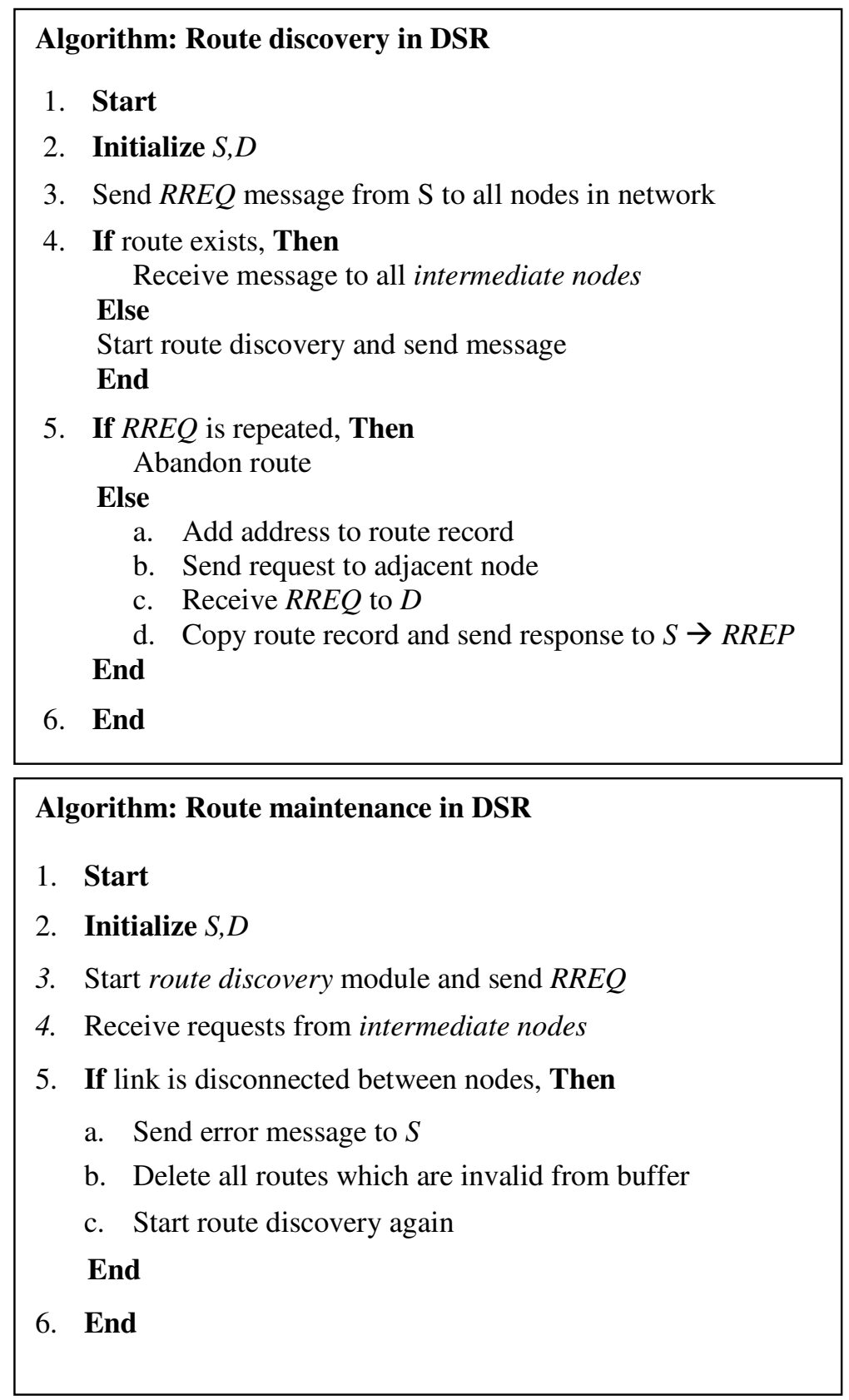

A general architecture for DSR considering the procedure of route discovery and route maintenance is given in figure 2 below. Considering the route discovery module, the process starts when source node (S) has to send a message to destination node (D) in a specified route. S floods the network with the RREQ, when the adjacent nodes receive the RREQ it checks whether the request is repeated, if repeated then, the adjacent node ignores the request. If the RREQ is not repeated, then it sends an RREP to $S$ (In this case the adjacent node acts as a destination node D) having the route information. When $\mathrm{S}$ receives the RREP it consecutively sends the message in the route specified by RREP. 


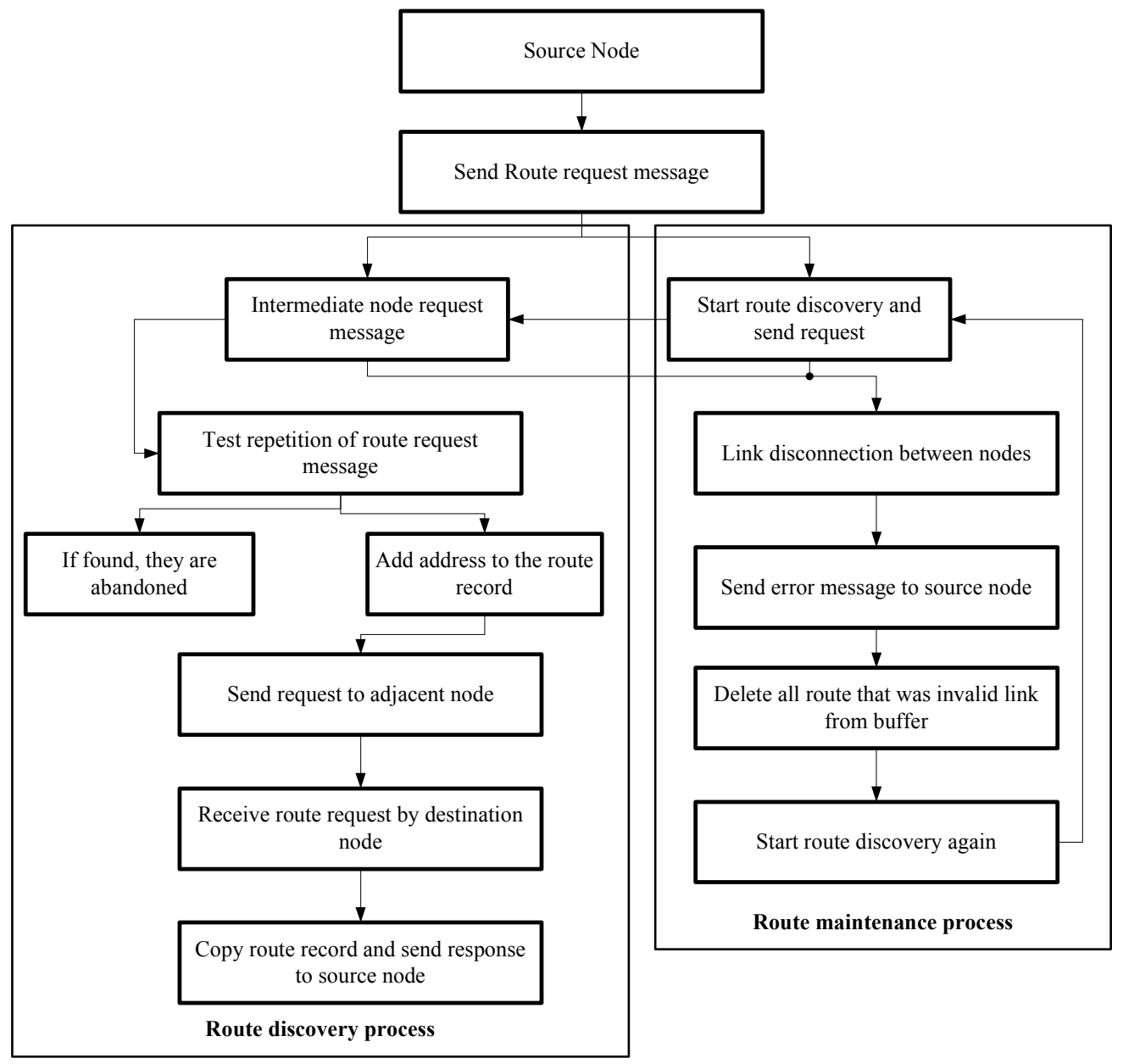

Figure 2: General architecture of DSR routing protocol

Considering the process of route maintenance, if the link between $\mathrm{S}$ and $\mathrm{D}$ is disconnected, the respective node sends an error message RERR to $\mathrm{S}$. S receives RRER and consequently deletes all the routes with this invalid link and starts the route discovery process all over again. It is observed that the route discovery process discovers many routes from $\mathrm{S}$ to $\mathrm{D}$, but the route chosen would not necessarily be with a minimum hop, hence more energy is consumed during data transmission. To improve the energy efficiency, a modification is performed in the conventional DSR as shown below.

\section{RESUlTS AND DisCUSSIONS}

The simulation results obtained in this experiment is analyzed by mainly three metrics, namely energy consumption, maximum throughput and delay. A brief explanation for each of the metric is given below as follows.

\subsection{ENERGY CONSUMPTION}

Average energy consumption is computed as the total consumed energy considering all nodes by number of nodes. It is mathematically represented as shown below.

$$
\text { Average energy }=\frac{\text { Total energy consumed by all nodes }}{\text { number of nodes }}
$$


A comparative analysis is performed with respect to energy consumption considering the DSDV, DSR, AODV and modified DSR. It is observed from figure 3 that the modified DSR has a stable and reduced energy consumption compared to DSDV, AODV and DSR.

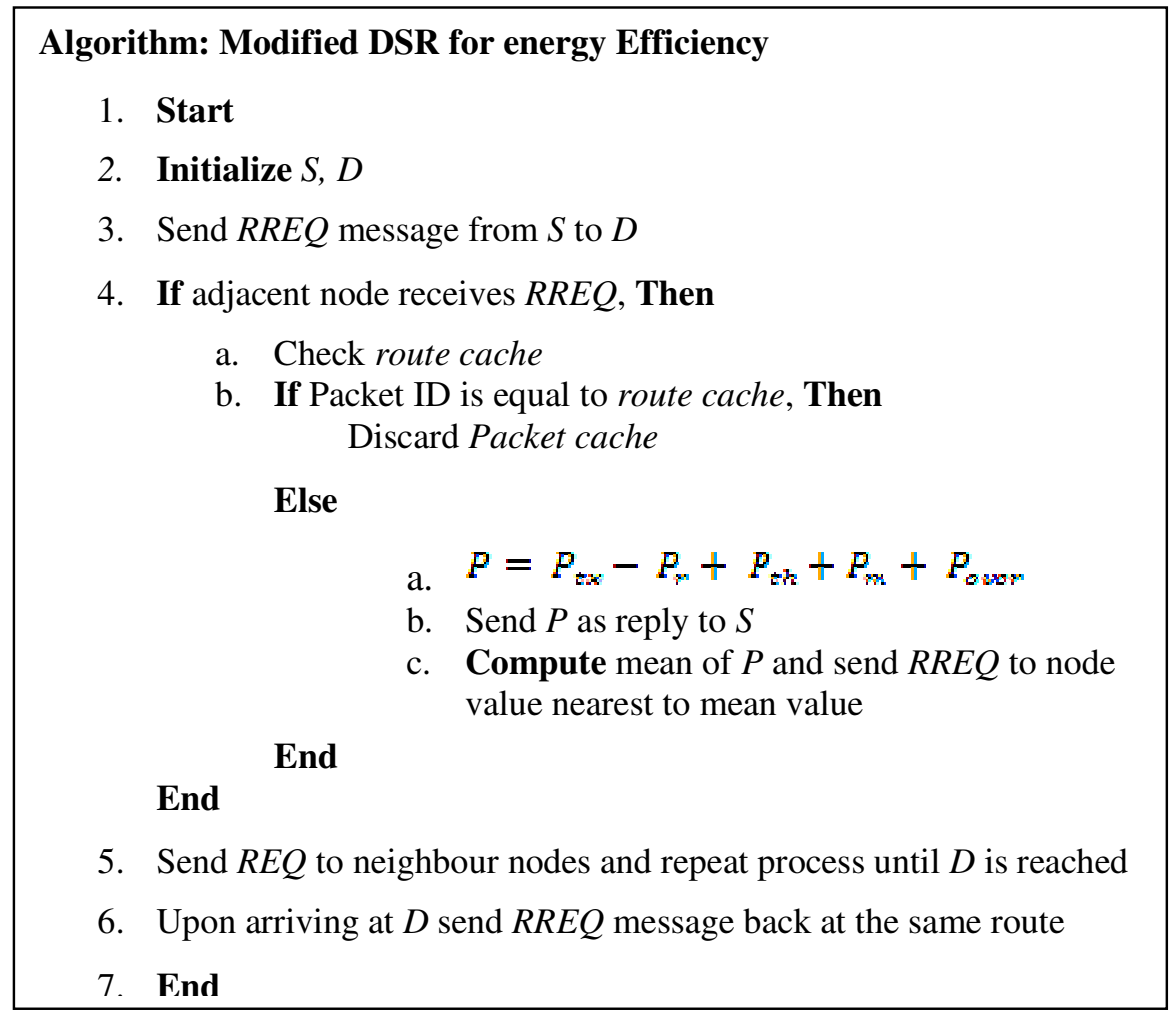

\subsection{MAXIMUM THROUGHPUT}

The network throughput is defined as the ratio of average rate of successfully delivered message to communication channel. It is mathematically defined as follows.

$$
\text { maximum throughput }=\frac{\text { Average rate of sucessfully delivered message }}{\text { communication channel }}
$$

A comparative analysis is performed with respect to maximum throughput considering the DSDV, DSR, AODV and modified DSR. It is observed from figure 4 that the modified DSR has a stable throughput compared to DSDV, AODV and DSR.

\subsection{DELAY}

The end to end delay is defined as an average time from the start of transmission time at source node to packet delivery at destination node. The delay is caused due to various reason, such as Buffering of data packets during route discovery, queuing at interface, propagation time, transfer time and retransmission delay. Figure 5 shows the delay caused in different protocols which is compared with the proposed modified DSR. Experimental results shows that the Proposed modified DSR has the minimum delay when compared to DSDV, AODV and DSR. 
International Journal of Ad hoc, Sensor \& Ubiquitous Computing (IJASUC) Vol.7, No.5, October 2016

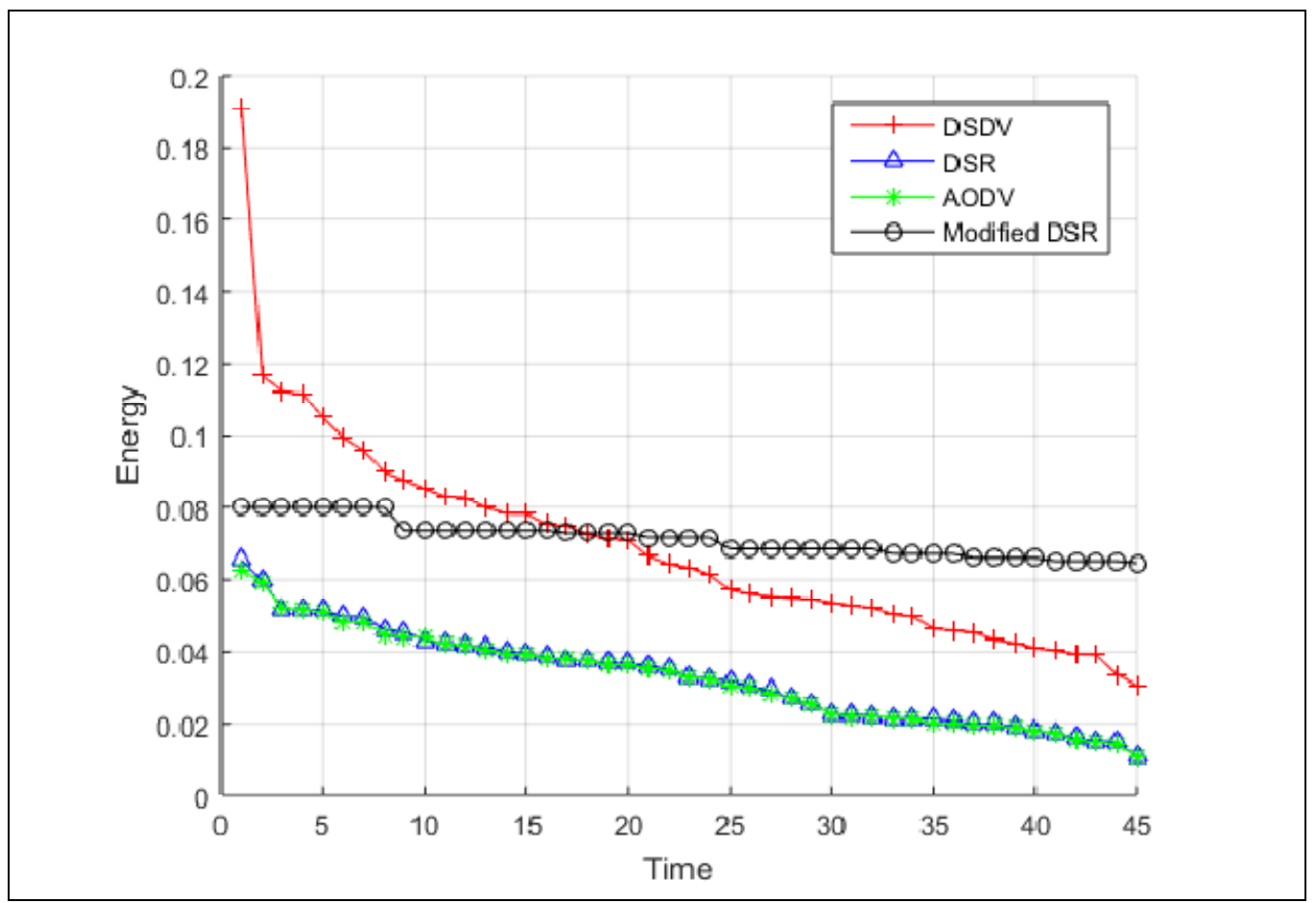

Figure 3: Comparative analysis on energy consumption for proposed modified DSR with DSDV, AODV and DSR

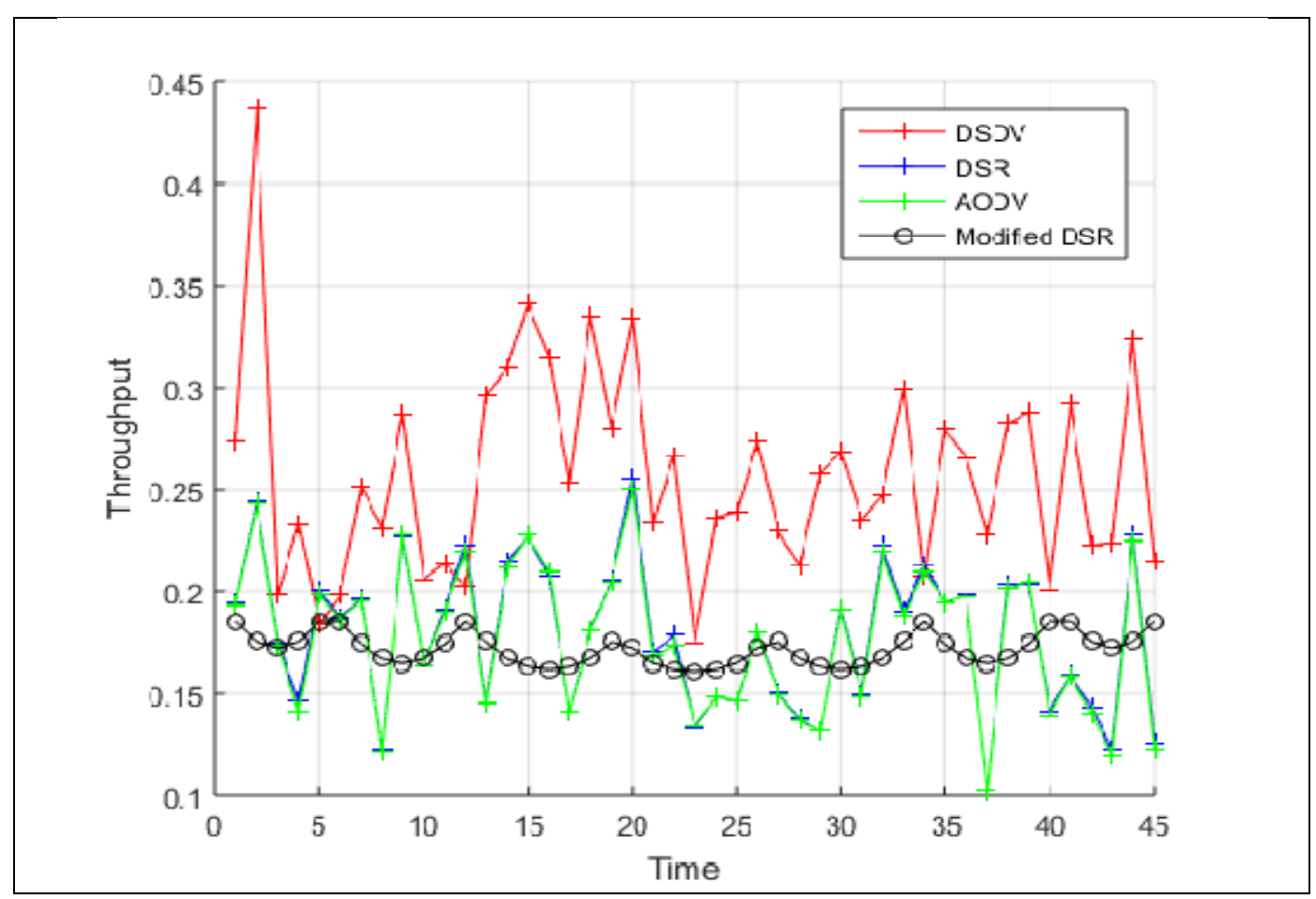

Figure 4: Comparative analysis on maximum throughput for proposed modified DSR with DSDV, AODV and DSR 


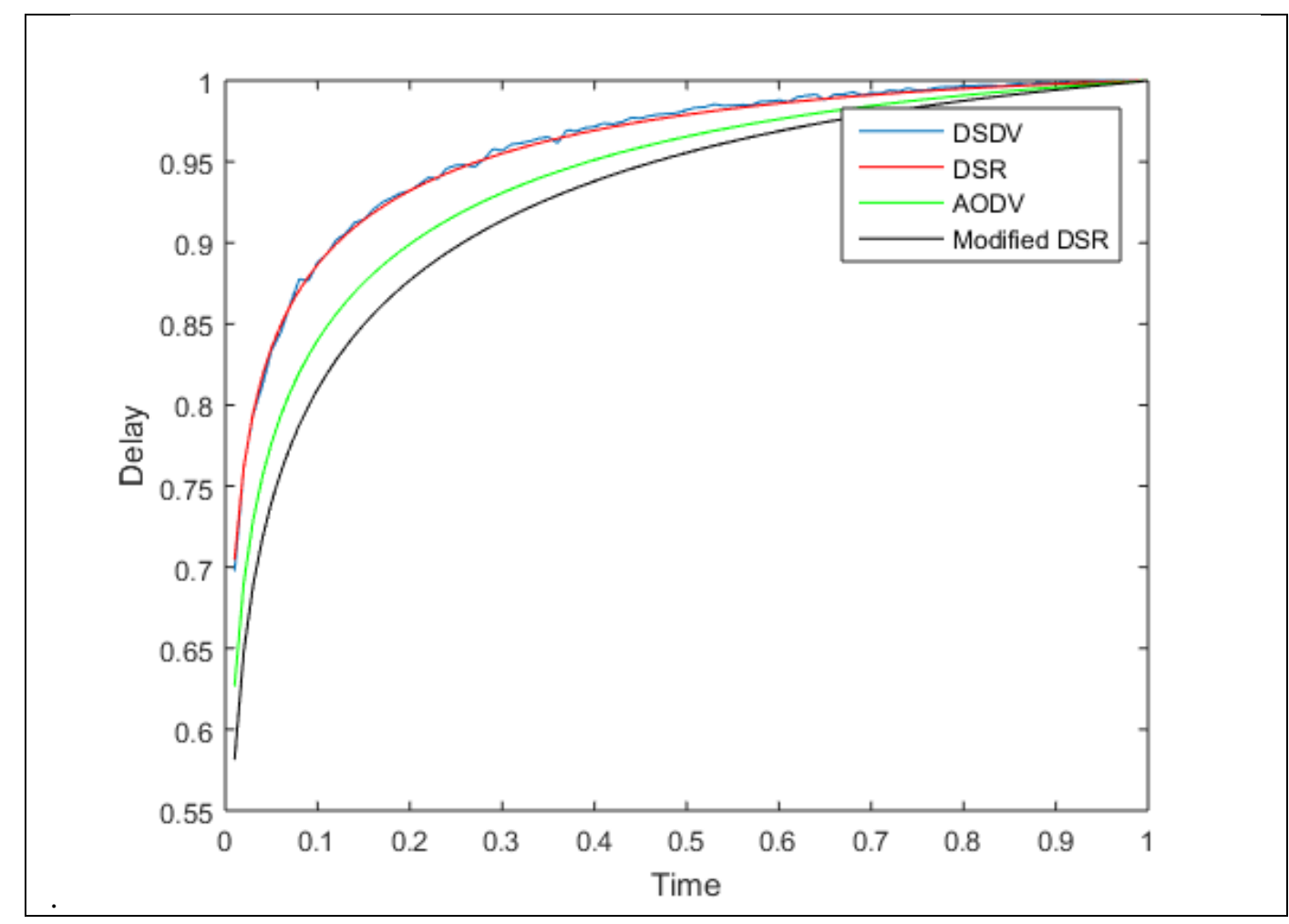

Figure 5: Comparative analysis on delay for proposed modified DSR with DSDV, AODV and DSR

\section{CONCLUSION}

In this experiment, an energy efficient routing protocol is proposed which is a modification of DSR. A comparative analysis is performed with respect to energy consumption, maximum throughput and delay. The routing protocols considered for this comparative analysis are DSDV, AODV and DSR. It is observed that the proposed modified DSR has stable and reduced energy consumption when compared with other standard routing protocols. The modified DSR also shows an improved rate of maximum throughput with reduced delay when compared to other standard routing protocol considered in this experiment. Analysis from the simulation results show an increased efficiency in energy consumption of $10 \%$, the fluctuation in the maximum throughput is decreased by $6 \%$ and a reasonable variation in delay as compared to other routing protocols.

\section{REFERENCES}

[1] David B. Johnson, (1994) "Routing in Ad Hoc Networks of Mobile Hosts". Proceedings of the Workshop on Mobile Computing Systems and Applications, IEEE Computer Society, Santa Cruz, CA, pp. 158-163.

[2] Esseghir Moez., (2011) "An implementation of energy-based dynamic routing for wireless sensor networks." In International Workshop on Systems, Signal Processing and their Applications, WOSSPA.

[3] Srinivas, K., A. Nagaraju, S. Ramachandram, and G. Narsimha, (2010) "Performance evaluation of routing protocols in static and dynamic ad-hoc networks based on energy consumption." In 
Information Technology for Real World Problems (VCON), 2010 Second Vaagdevi International Conference on, pp. 47-51. IEEE,

[4] Cisco Networking Academy, (2014) "Introduction to Routing Dynamically", Cisco Press

[5] Barati, M., Atefi, K., Khosravi, F. and Daftari, Y.A., (2012), "Performance evaluation of energy consumption for AODV and DSR routing protocols in MANET". In Computer \& Information Science (ICCIS), 2012 International Conference on (Vol. 2, pp. 636-642). IEEE.

[6] Kunavut, K., 2014, "September. Performance and comparative analysis of energy consumption for mobile ad hoc routing protocols". In Communications and Information Technologies (ISCIT), 2014 14th International Symposium on (pp. 76-79). IEEE.

[7] Alam, S., De, D. and Ray, A., (2015), “Analysis of Energy Consumption for IARP, RIP and STAR Routing Protocols in Wireless Sensor Networks". In Advances in Computing and Communication Engineering (ICACCE), 2015 Second International Conference on (pp. 11-16). IEEE.

[8] Tavosian, E., Bag-Mohammadi, M. and Moghadam, R.A., (2012)," Using network coding to improve energy consumption in AODV routing protocol." In 2012 18th Asia-Pacific Conference on Communications (APCC) (pp. 1002-1006). IEEE.

[9] Kajikawa, H., Lin, I.T. and Sasase, I., (2012), "Grid-based routing protocol using cell rotation to reduce packets latency and energy consumption in wireless sensor networks." In 2012 IEEE Consumer Communications and Networking Conference (CCNC) (pp. 699-701). IEEE.

[10] Weng, C.C., Chen, C.W. and Ku, C.J., (2011), “A minimum transmission energy consumption routing protocol for user-centric wireless networks." In System Integration (SII), 2011 IEEE/SICE International Symposium on (pp. 1143-1148). IEEE.

[11] Qin, H., Zhong, X. and Xiao, Z., (2011), "Balanced energy consumption and cluster-based routing protocol”. In 2011 9th IEEE International Conference on Control and Automation (ICCA) (pp. 686691). IEEE.

[12] Friginal, J., de Andrés, D., Ruiz, J.C. and Gil, P., (2011), "Using performance, energy consumption, and resilience experimental measures to evaluate routing protocols for ad hoc networks." In Network Computing and Applications (NCA), 2011 10th IEEE International Symposium on (pp. 139-146). IEEE.

[13] Zhang, J.H., Peng, H. and Shao, F.J., (2011), "Energy consumption analysis of MANET routing protocols based on mobility models." In Fuzzy Systems and Knowledge Discovery (FSKD), 2011 Eighth International Conference on (Vol. 4, pp. 2275-2280). IEEE.

[14] Brar, G.S., Rani, S., Chopra, V., Malhotra, R., Song, H. and Ahmed, S.H., "Energy Efficient Direction Based PDORP Routing Protocol For Wireless Sensor Networks."

[15] Pan, L., (2015), “An improved the DSR routing protocol in mobile ad hoc networks." In Software Engineering and Service Science (ICSESS), 2015 6th IEEE International Conference on (pp. 591594). IEEE.

[16] Pathak, G.R., Patil, S.H., Rana, A.D. and Suralkar, Y.N., (2014), "Mathematical model for routing protocol performance in NS2: Comparing DSR, AODV and DSDV as example." In Wireless Computing and Networking (GCWCN), 2014 IEEE Global Conference on (pp. 184-188). IEEE.

[17] Ahuja, R., Ahuja, A.B. and Ahuja, P., (2013), "Performance evaluation and comparison of AODV and DSR routing protocols in MANETs under wormhole attack." In Image Information Processing (ICIIP), 2013 IEEE Second International Conference on (pp. 699-702). IEEE.

[18] Tambuwal, A.B., Noor, R.M. and Michael, O., (2013), "Improvement of DSR routing protocol using TTL-based scheme in Mobile Ad Hoc Networks." In RFID-Technologies and Applications (RFIDTA), 2013 IEEE International Conference on (pp. 1-6). IEEE.

[19] D. Johnson, Y. Hu and D.Maltz, (2007), "The Dynamic Source Routing Protocol (DSR) for Mobile Ad hoc networks for IPv4", The IETF Trust (2007)

[20] Istikmal, (2013), "Analysis and evaluation optimization dynamic source routing (DSR) protocol in mobile AdHoc network based on ant algorithm”, 2013 IEEE. 


\section{AuTHORS}

JIJESH J J is currently an Assistant Professor in the Electronics and Communication Department of Sri Venkateshwara College of Engineering, Bangalore. He received the B.Tech. degree in Electronics and Communication Engineering from Kannur University in 2006, and earned M.Tech. degree in Electronics from VTU in 2010 and presently pursuing the Ph.D. degree in VTU. He has more than 15 publications in his credit. He has served as reviewer for IEEE Conferences.

SHIVASHANKAR is a Professor and HOD in the Electronics and Communication Department of the Sri Venkateshwara College of Engineering, Bangalore. He received the B.E. degree in Electronics and Communication Engineering from Mysore University in 2000, and the M.Tech. degree in computer science engineering from Mysore University in 2005 and earned his Ph.D. degree in wireless communication from VTU. He has published more than 25 publications and guiding research in different universities. He has been associated with numerous IEEE Conferences as Technical Program Committee and served as session chair and reviewer for

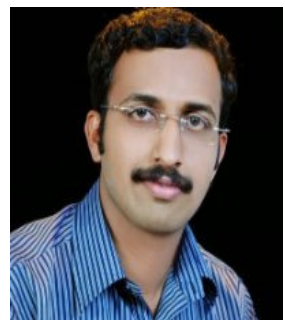
IEEE Conferences.

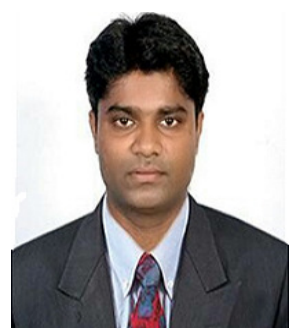

